Article

\title{
Design of Cyclone Separator Critical Diameter Model Based on Machine Learning and CFD
}

\author{
Donggeun Park ${ }^{1}\left(\mathbb{D}\right.$ and Jeung Sang Go ${ }^{2, *}$ (i) \\ 1 Department of Advanced Materials and Parts of Transportation Systems, Pusan National University, \\ Busan 46241, Korea; dgpark94@pusan.ac.kr \\ 2 School of Mechanical Engineering, Pusan National University, Busan 46241, Korea \\ * Correspondence: micros@pusan.ac.kr
}

Received: 3 November 2020; Accepted: 21 November 2020; Published: 23 November 2020

\begin{abstract}
In this paper, the characteristics of the cyclone separator was analyzed from the Lagrangian perspective for designing the important dependent variables. The neural network network model was developed for predicting the separation performance parameter. Further, the predictive performances were compared between the traditional surrogate model and the developed neural network model. In order to design the important parameters of the cyclone separator based on the particle separation theory, the force acting until the particles are separated was calculated using the Lagrangian-based computational fluid dynamics (CFD) methodology. As a result, it was proved that the centrifugal force and drag acting on the critical diameter having a separation efficiency of $50 \%$ were similar, and the particle separation phenomenon in the cyclone occurred from the critical diameter, and it was set as an important dependent variable. For developing a critical diameter prediction model based on machine learning and multiple regression methods, unsteady-Reynolds averaged Navier-Stokes analyzes according to shape dimensions were performed. The input design variables for predicting the critical diameter were selected as four geometry parameters that affect the turbulent flow inside the cyclone. As a result of comparing the model prediction performances, the machine learning (ML) model, which takes into account the critical diameter and the nonlinear relationship of cyclone design variables, showed a $32.5 \%$ improvement in R-square compared to multi linear regression (MLR). The proposed techniques have proven to be fast and practical tools for cyclone design.
\end{abstract}

Keywords: cyclone separator; computational fluid dynamics (CFD); machine learning; unsteady RANS; critical diameter

\section{Introduction}

Cyclone separators with cheap and high separation performance have been mainly used to reduce the emissions from industrial and manufacturing processes. The cyclone separates the contaminant particles by the turbulence flow. The cyclone flows have the outer flow and inner flow. The outer flow rotates along the wall to the bottom of the dust container and an inner flow is reversed at the end of the dust container and discharged to the cyclone outlet as shown Figure 1. The centrifugal force pushes the particle to the wall (the outer flow region) and the drag force pushes the particle to the cyclone center (inner flow region). In other words, when the centrifugal force acting on the particles is greater than the drag force, the particles are trapped into the cyclone duct container. In order to increase the separation performance of the cyclone, it is necessary to design the cyclone shape so that the centrifugal force acts more than the drag force on the particles with the smallest diameter possible. The turbulent behavior of cyclone is primarily influence by the size of the cyclone shape. 


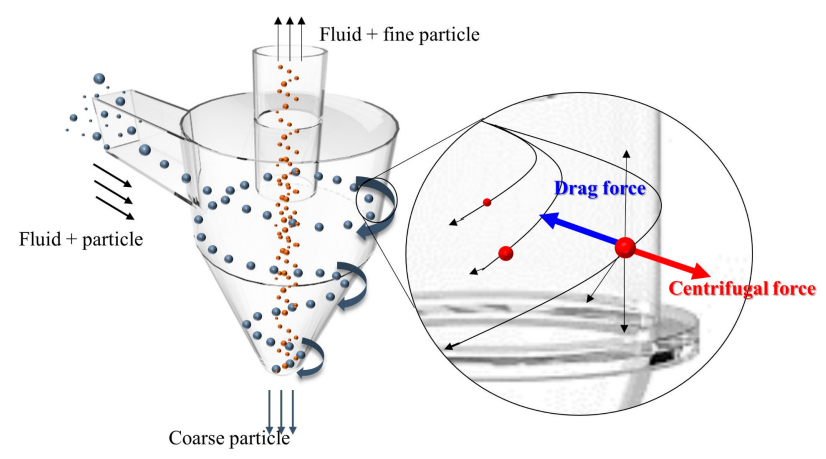

Figure 1. Geometry of the cyclone separator.

Therefore, many studies have been conducted over the past decades to optimize the separation performance according to the shape of the cyclone. Early researchers developed an empirical equation based on the experimental results about the particle separation changing the various cyclone shape and the equation derived by the physic law [1-3]. However, since the empirical equations are based on experimental values with uncertainty, the reproducibility of the results is poor.

With the rapid development of computing speed and numerical analysis techniques, many studies were conducted to predict cyclone flow by solving Reynolds averaged Navier-Stokes equations based on commercial computational fluid dynamic (CFD) codes. The CFD studies have investigated the effect on separation performance by independently setting several cyclone geometric design variables [4-10]. For example, the separation efficiency and pressure drop were evaluated according to the inlet shape without changing other shapes by using CFD [6]. The cyclone performance investigated according to the relationship between the cyclone outlet shape and the shape of the dust container $[8,9]$. Moreover, the CFD method was used to analyze the internal flow that cannot obtain the information through experiments. However, there is a possibility to obtain the local optimization due to considering the independently geometric design variables. In addition, it takes a lot of computational cost to obtain the cyclone separation performance according to various shapes by using CFD.

In order to solve the local optimization problem and computing cost problem, the method combining CFD and surrogate modeling has been applied for the relationship between cyclone shapes and separation performance [11-16]. For example, the surrogate models such as artificial neural network (ANN), response surface methodology (RSM) and group method of data handling (GMDH) algorithm showed the reasonable predictive performance, and optimum design was performed by applying optimization algorithms such as genetic algorithms (GA) [13]. However, the most optimization studies omitted the analysis of the cause of the optimal separation performance. For analyzing the optimization results, the tangential velocity contour, and the velocity distribution before and after optimization was compared based on the particle separation theory from Euler's point of view [13,14]. However, since the force acting on the particles differs according to the rotational trajectory, it is more appropriate to analyze it according to the trajectory position rather than to analyze it from the Euler perspective. In other words, it is necessary to analyze the force acting according to the particle trajectory in the cyclone from the Lagrangian perspective. By analyzing the cyclone separation performance from the Lagrangian point of view, the verified cyclone separation performance parameter can be newly considered as a dependent variable of the cyclone design.

This study has two purposes, (1) to analyze the characteristics of cyclones from the Lagrangian perspective by using CFD for designing important dependent variables that are different from previous studies, (2) to develop a machine learning prediction model of the obtained dependent variables, and (3) to compare the prediction performance with the machine learning model and traditional surrogate model. This study identifies more rational dependent variables for cyclone design. In addition, it is possible to propose a fast and reliable process.

The flow chart of this study is summarized as shown in Figure 2. First, the characteristics of cyclones analyzed from the Lagrangian perspective in order to obtain meaningful dependent variables. 
Next, a CFD data set about the dependent variables is generated to develop a machine learning model. The data sets are created by a design space with various combinations using the design of experiment (DoE) method. Then, the machine learning model for cyclone separation performance are developed by the CFD data set. Finally, the developed model evaluates the predictive performance compared to the traditional surrogate model, MLR.

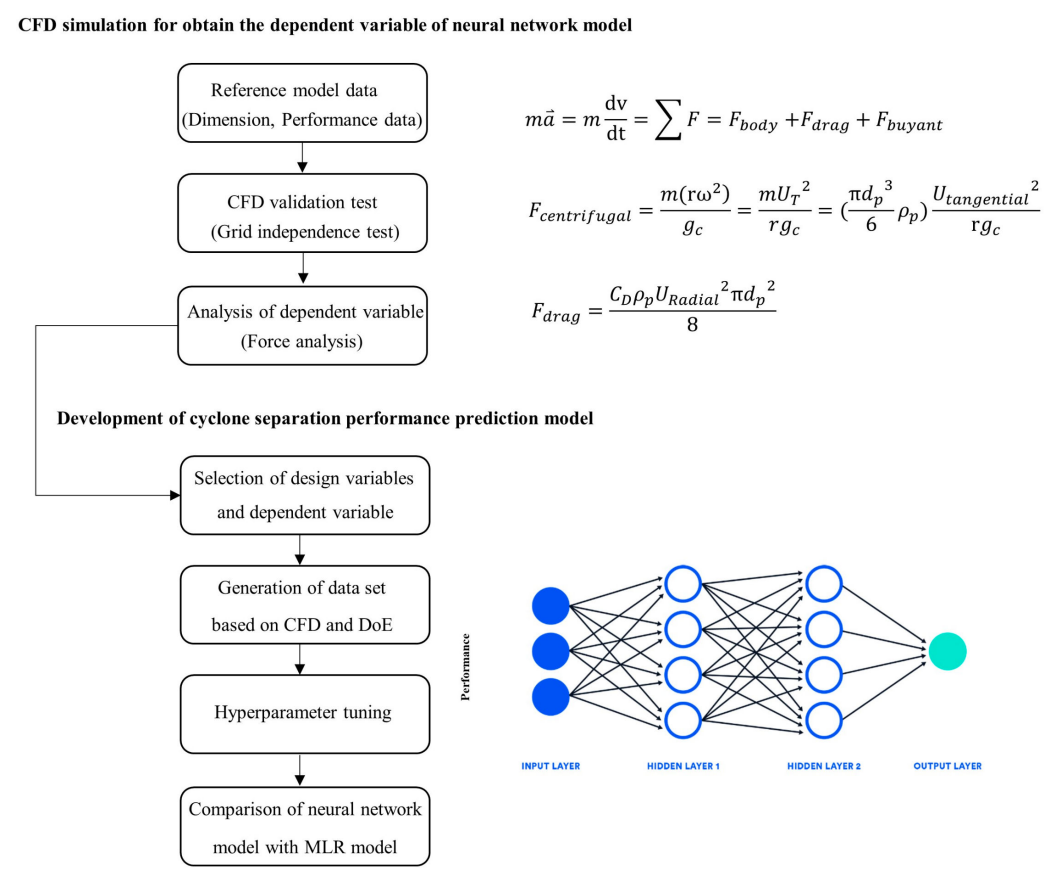

Figure 2. Research flow chart.

\section{Research Methods}

\subsection{Governing Equation for Numerical Simulation}

The numerical simulation was applied to obtain the dependent variable of the separation performance for machine learning algorithm. The cyclone flow has strong three-dimensional rotational turbulence. For analyzing the cyclone flow, the 3D Reynolds averaged Naiver-Stokes equation are solved based on finite volume method. The Equation (1) represents the RANS equation.

$$
\frac{\partial \bar{u}_{\mathrm{i}}}{\partial \mathrm{t}}+\bar{u}_{j} \frac{\partial \bar{u}_{\mathrm{i}}}{\partial \mathrm{x}_{\mathrm{j}}}=-\frac{1}{\rho} \frac{\partial \bar{p}}{\partial \mathrm{x}_{\mathrm{i}}}+\frac{\partial^{2} \bar{u}_{\mathrm{i}}}{\partial \mathrm{x}_{\mathrm{j}} \partial \mathrm{x}_{\mathrm{j}}}-\frac{\partial}{\partial \mathrm{x}_{\mathrm{j}}} u_{i}^{\prime} u_{j}^{\prime}
$$

where the $p$ is pressure. the velocity components are decomposed into the mean velocity, $\bar{u}_{i}$ and fluctuating velocity, $u_{i}^{\prime}$, respectively. The $u_{i}^{\prime}$ is Reynolds stress. The additional process of Reynolds stress is required to solve the RANs equation. The various turbulence models have been used for solve the Reynolds stress term [17]. Appropriate turbulence models must be applied to obtain high-accuracy numerical analysis results. The turbulence models have been successfully applied in many industrial fields. For detailed equations and explanations on the turbulence model, reference is made to the length limitation of this paper $[17,18]$.

The behavior of fluid and solid particles was simulated complementarily for investigating the dynamic behavior of particle in cyclone. In order to design rationally the cyclone separation performance parameters, which is one of the objectives of this study, it is necessary to track the dynamic 
behavior of solid particles based on the Lagrangian method rather than the Euler method. The Equation (2) represents the particle trajectory equilibrium equation [17].

$$
\frac{d \vec{u}_{p}}{d t}=\frac{\left(\vec{u}_{k}+\vec{u}_{k}^{\prime}-\vec{u}_{p}\right)}{\tau}+\frac{\vec{g}\left(\rho_{p}-\rho\right)}{\rho_{p}}+\mathrm{F}
$$

where the term $\frac{\left(\vec{u}_{k}+u_{k}^{\prime}-\vec{u}_{p}\right)}{\tau}$ is the drag force per unit particle mass, $\vec{u}_{p}$ is the particle velocity, $\vec{u}_{k}$ is the flow phase velocity, and $F$ is an additional acceleration (force/unit particle mass). The $\vec{u}_{k}^{\prime}$ as the term of turbulence transport equation influences the behavior of the particles. $\tau$ is particle relaxation time. $\tau$ is as follow in Equation (3):

$$
\tau=\frac{18 \mu}{d_{p}^{2} \rho_{P}} \frac{R e_{p}}{24} C_{D}
$$

where the $d_{P}$ is the particle diameter, $\rho_{P}$ is density of particle, $\mu$ is the density of fluid, $R e_{p}$ is the particle Reynolds number, and $C_{D}$ is the drag coefficient. In this study, the commercial CFD code ANSYS 16.1 was used to solve Equations (1) and (2). The computational domain of cyclone is used as a cutcell type as shown Figure 3. The cyclone dimension of reference was cited for validating CFD results [19]. The near-wall treatment was achieved by using scalable wall functions considering the grid refinement with $\mathrm{y}^{+}<11$. The Table 1 shows the boundary conditions for numerical analysis applied in this study. The simulation time is set as 1.5 second considering the physic time. For CFD simulation, the SIMPLE algorithm, PRESTO! alogorithm. Second order upwind scheme were used for pressure term, pressure-velocity term, and turbulence kinetic and dissipation and momentum term, respectively. The criteria of residual values of the turbulence equation and other equation for assessing CFD convergence were set as $10^{-6}$ and $10^{-4}$.

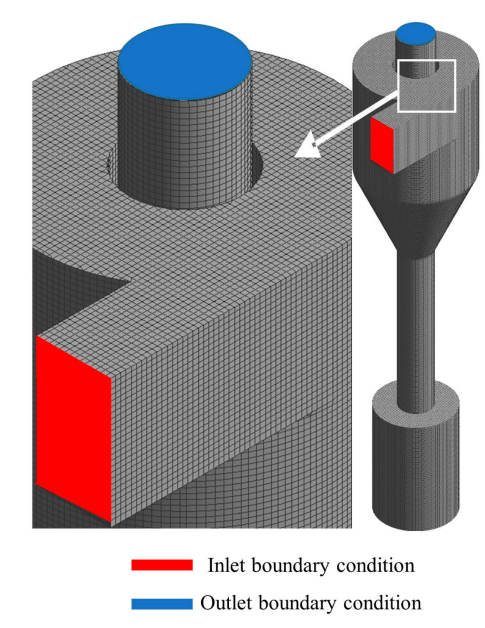

Figure 3. Computational domain of cyclone separators.

Table 1. Boundary condition for computational fluid dynamics (CFD).

\begin{tabular}{cc}
\hline Boundary Condition & Values \\
\hline Inlet velocity & $800\left(\mathrm{~m}^{3} / \mathrm{h}\right)$ \\
Pressure drop & $1 \mathrm{~atm}$ \\
Time step size & $0.001 \mathrm{~s}$ \\
Number of time step & 1500 \\
\hline
\end{tabular}

\subsection{Machine Learning Algorithm}

The CFD simulations with combinations of various geometric design variables are time consuming. A machine learning model for cyclone separation performance has been developed for solving the time 
cost problem of cyclone design. The developed model can predict fast separation performance changing the various design combinations. The separation performance model was developed using the back propagation neural network model among machine learning algorithms. The neural network model predicts the output variable according to the new input variable by giving nonlinear characteristics to the relationship between the input design variable and the output variable. The structure of the neural network consists of several hidden layers between input and output variables. The layer consists of various nodes, and the node converts the linear combination of input variables into a sigmoid nonlinear form as shown in Equations (4) and (5).

$$
\begin{gathered}
\mathrm{y}_{j}^{(\mathrm{k})}=\mathrm{b}_{0}+\sum_{\mathrm{i}=1}^{\mathrm{n}} \mathrm{w}_{\mathrm{i}} \mathrm{x}_{\mathrm{i}} \\
\mathrm{y}_{\mathrm{j}_{\text {_out }}(\mathrm{k})}=\frac{1}{1+\exp \left(-\mathrm{y}_{j}^{(\mathrm{k})}\right)}
\end{gathered}
$$

where $\mathrm{k}$ is layer number, $j$ is node number, and $\mathrm{w}_{\mathrm{i}}$ is weight. The input variables are transferred to the hidden layer and calculated until the end of the output. Then, the weight of all nodes are updated repeatedly so that the error with the true value is minimized. This is called backpropagation process. That is, the parameters such as learning rate, epoch, batch size, and number of hidden layers etc. must be optimized to make the minimum difference value between the true value and prediction value.

In this study, input variables of neural network model were set as the four cyclone geometrical variables to make a model for predicting cyclone separation performance. It was confirmed that four geometric variables out of many geometries have a great influence on the cyclone separation performance [12]. The combination of design variables for developing the neural network model was created based on the design of experiment (DOE). The combination is called as data set. The DOE enables to create a design area that a lot of information can be obtained with little data. The range of input design variables is shown in the Table 2. The minimum and maximum bounds were created in a range that interference does not occur between shapes. The total number of data set is 100 . The data set is generated by using DOE sampling method. In general, the data set consisted of a training set, a validation set, and a test set, with each percentage set to $70 \%, 10 \%$, and $20 \%$, respectively [13]. Since the train set and test set have the different variable combinations, it is possible to evaluate the predictive performance of the generalized model. The validation set is a set that checks whether over fitting occurs in the process of updating weights. The Figure 4 shows the design space of the train set and test set for the cyclone shape variables used in this study. In the Figure 4, the x1, x2, x3, and $\mathrm{x} 4$ is the four cyclone geometrical variables. In the Figure 4 , the line represents the normalization curve for the distribution of values of a particular variable in 100 data sets. The dots represent the correlation between the variables as a combination. For example, the upper left of Figure 4 shows the normalization curve for the $x$-axis range of the $x 1$ variable. That is, the distribution of the train set and the test set is different. The upper right of Figure 4 shows the combination between the $x 1$ and $x 4$ variables in the data set. It can be seen that the combination of train dataset and test dataset is different. Because the design space of the train set and the test set are different, the generalized performance tests can be performed. All BPNN calculations were carried out using PYTHON 3.6. 

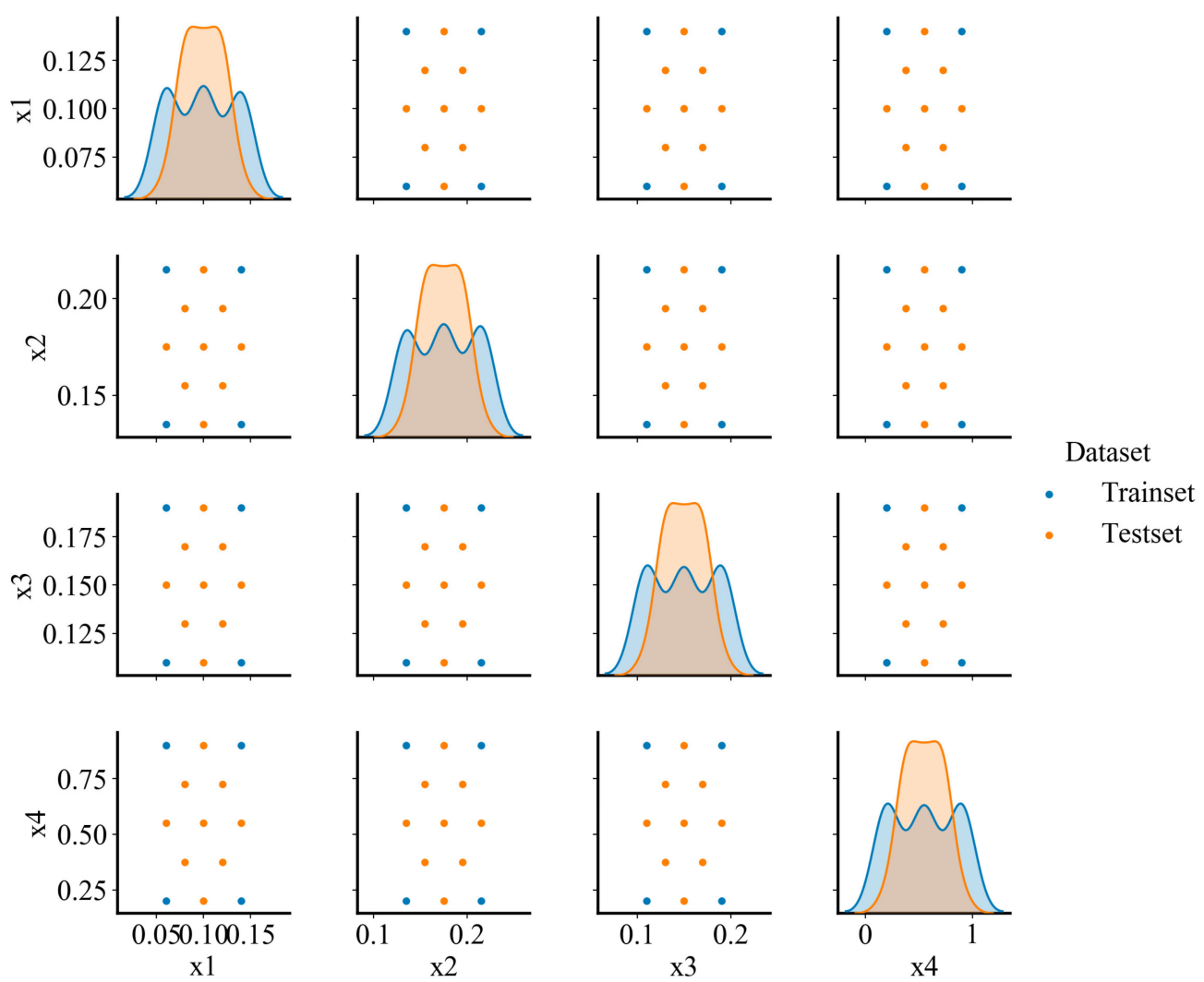

Figure 4. Design space for dataset; $x 1, x 2, x 3$, and $x 4$ is design variable of cyclone.

Table 2. Range of design variable of cyclone.

\begin{tabular}{ccc}
\hline Boundary Condition & Min $\left(\mathbf{x} / \mathbf{D}^{\mathbf{1}}\right)$ & $\mathbf{M a x}\left(\mathbf{x} / \mathbf{D}^{\mathbf{1}}\right)$ \\
\hline Outlet diameter & 0.275 & 0.475 \\
Inlet width & 0.15 & 0.35 \\
Inlet height & 0.3375 & 0.5375 \\
Cone length & 0.5 & 1.95 \\
\hline \multicolumn{2}{c}{}
\end{tabular}

\section{Results}

\subsection{CFD Simulation Result for Validation}

To achieve the purpose of this study, it is essential to validate the use of CFD. The experimental results and cyclone dimensions were cited $[19,20]$. The geometric schematic diagram and dimension were represented as Figure 5 and Table 3, respectively. The mesh-independence test not only helps the efficient use of computing cost, but also obtains a numerically optimized computational domain [21]. To verify the validity of the CFD, a mesh-independence test was performed by calculating the particle separation efficiency. The particle separation efficiency refers to the ratio of the total number of particles injected at the inlet and the number of particles collected in the dust container. The discreet phase modeling (DPM) was used to calculate the separation efficiency [17]. The total number of particles are $10^{4}$. The particle density is $2770 \mathrm{~kg} / \mathrm{m}^{3}$. The particle size distribution is divided to 10 class based on the Rosin-Rammler theory as Equation (6).

$$
Y_{D}=e^{-(d / \bar{d})^{n}}
$$

where the $\bar{d}$ is the mean diameter, and the $n$ is the diffusion parameter. For simulation, the $\mathrm{d}$ and $\mathrm{n}$ are defined to $5 \mu \mathrm{m}$ and $3.5 \mu \mathrm{m}$, respectively. Moreover, the distribution of particle diameters is set from 1 
to $10 \mu \mathrm{m}$. The simulation results by decreasing mesh size were compared with the cited experimental data [19]. The three mesh types as coarse, fine, and finest were used for mesh-independent test. The total number and mesh size of coarse types are $5.35 \times 10^{5}$ and $100 \mathrm{~mm}$, respectively. The total number and mesh size of fine types are $5.81 \times 10^{6}$ and $6.5 \mathrm{~mm}$, respectively. The total number and mesh size of coarse types are $9.58 \times 10^{6}$ and $3.5 \mathrm{~mm}$, respectively. The near-wall treatment was achieved by using scalable wall functions considering the grid refinement with $\mathrm{y}^{+}<11$. The growth from the wall is at a ratio of 1.5. The CFD results by three grid types were compared with the experimental data as Table 4. As the mesh size decreases, the numerical values converged. The error between the CFD results and the referenced experimental results was within $2 \%$. The grid size of fine type mesh was $6.5 \mathrm{~mm}$. The fine type mesh was selected due to the numeric accuracy and computational cost in this study. Furthermore, the mesh quality check for the fine type mesh was performed as shown the Table 5. The quality checking results show that the averaged skewness is 0.177 which represents the reasonable accuracy of mesh shape and the averaged aspect ratio of the fine mesh is about 1.814. Therefore, the fine type mesh is acceptable. The selected grid size is used as an input condition of CFD analysis for neural network modeling.

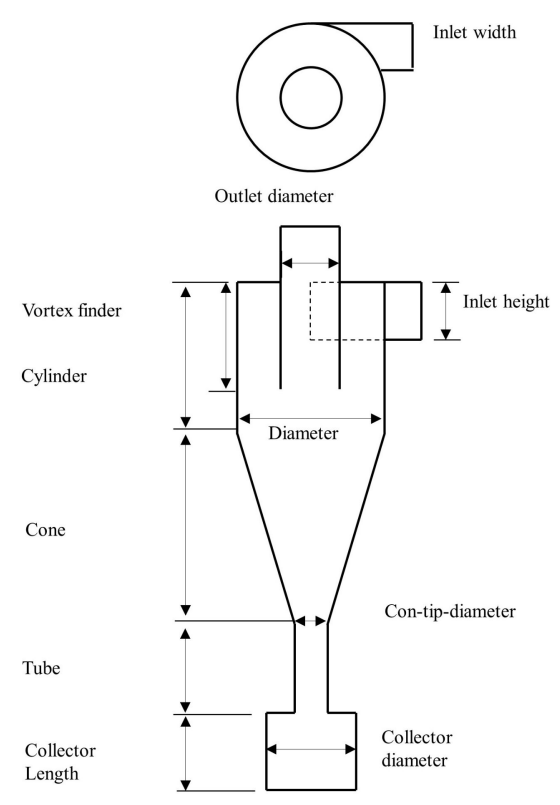

Figure 5. Geometric schematic diagram.

Table 3. Geometric parameter values.

\begin{tabular}{cc}
\hline Factors & Values $\left(\mathbf{x} / \mathbf{D}^{\mathbf{1}}\right)$ \\
\hline Outlet diameter & 0.375 \\
Inlet width & 0.25 \\
Inlet height & 0.4375 \\
Cone length & 1.225 \\
Cylinder length & 1.25 \\
Vortex finder length & 0.45 \\
Tube & 0.74 \\
Con-tip-diameter & 0.375 \\
Collector Length & 0.745 \\
Collector diameter & 0.735 \\
\hline
\end{tabular}

$\mathrm{D}^{1}$ is $0.4 \mathrm{~m}$. 
Table 4. Grid dependence test results.

\begin{tabular}{ccccc}
\hline Mesh Type & Coarse & Fine & Finest & Exp. [19] \\
\hline Separation efficiency & $52.21 \%$ & $84.42 \%$ & $84.35 \%$ & $83.5 \%$ \\
Error with Exp. [19] & $37.4 \%$ & $1.101 \%$ & $1.017 \%$ & - \\
\hline
\end{tabular}

Table 5. Mesh quality check results for the fine mesh type.

\begin{tabular}{cc}
\hline Mesh Type & Values \\
\hline Skewness average & 0.177 \\
Aspect ratio average & 1.814 \\
\hline
\end{tabular}

In addition, in order to select an appropriate turbulence model that can simulate a cyclone strong rotational flow, the results of the velocity distribution experiment [20] and the prediction results according to the turbulence model were compared. The experimental data and simulation data were compared with the results of the tangential velocity and axial velocity distribution at the certain locations $(\mathrm{Y}=0.77 \mathrm{D}, \mathrm{A}-\mathrm{A} \prime)$ as shown Figure 6 . The residual values of the turbulence equation and mass equation showed the under $10^{-6}$ and $10^{-4}$. In the Figure 6, the $x$ label is the distance from the center of the cyclone to the wall. When the $\mathrm{k}-\varepsilon$ model was used, it showed an abnormal tangential distribution near the wall. The reason for this prediction is that the $\mathrm{k}-\varepsilon$ model assumes anisotropic property for modeling the Reynolds stress term. When $\mathrm{k}-\varepsilon$ model is applied for cyclone flow analysis, the outer flow and inner flow can be captured incorrectly. In contrast, Reynolds stress model (RSM) predicted a velocity distribution similar to the experimental results. The RSM can properly simulate rotational flow through an isotropic assumption for Reynolds stress term. Therefore, in this study, the RSM was applied to capture the cyclone flow. The detailed equations and explanations on the RSM can refer the reference $[17,18]$.
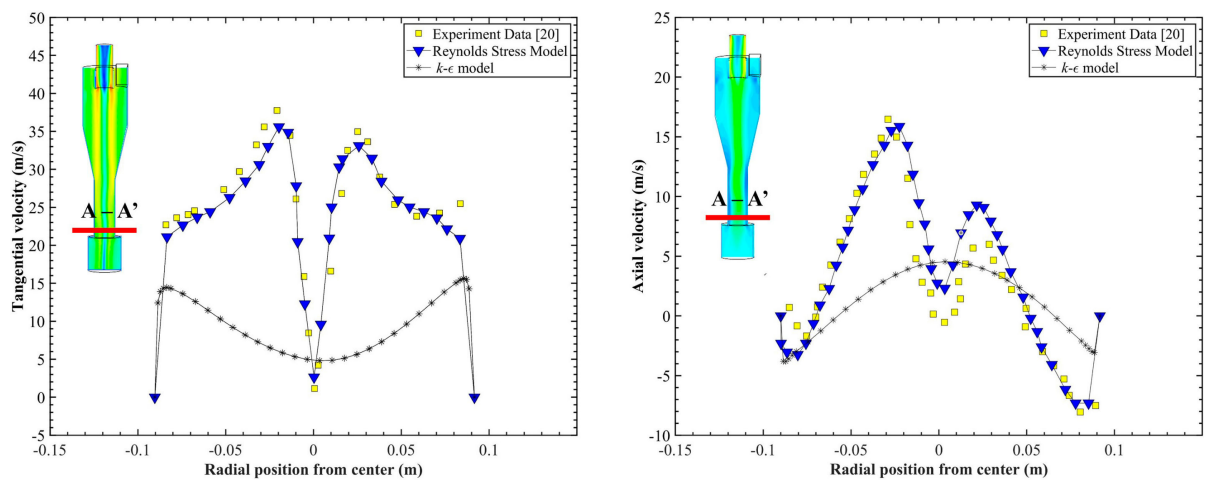

Figure 6. Comparison results of the velocity distribution experiment and the prediction results according to the turbulence model.

\subsection{CFD Simulation for the Dependent Variable of Cyclone}

The important dependent variables were designed by analyzing the characteristics of cyclones from the Lagrangian perspective. The force acting on the particles was calculated until the particles were separated by using ANSYS FLUET User Define Function code. The dynamic behavior results are very similar to the particle separation theory. The Figure 7 represents the force analysis results.

As shown Figure 7, In the case of $1 \mu \mathrm{m}$ particles, the drag acting on the particles is superior to the centrifugal force, so the particles enter the inner flow region and are discharged through the cyclone outlet. In the case of $1.5 \mu \mathrm{m}$, the centrifugal force and drag act similarly, resulting in rotational motion within the cyclone. Eventually, the drag force is slightly larger and the particles are rebound in the dust container. The $5 \mu \mathrm{m}$ particles have a larger centrifugal force than the drag force, so that the particles are collected in the dust container. 

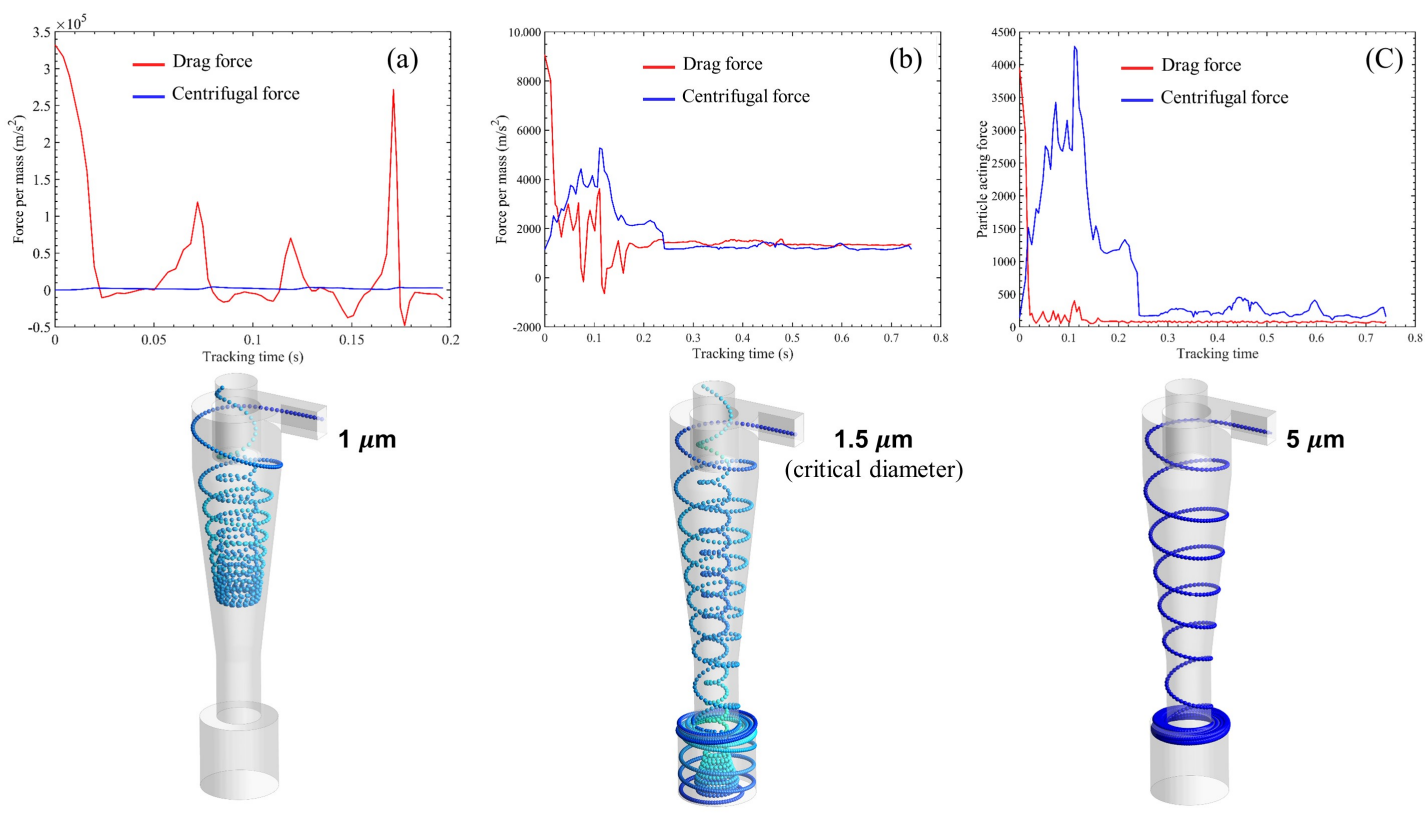

Figure 7. The force analysis acting on particle; (a) $1 \mu \mathrm{m}$ behavior, (b) $1.5 \mu \mathrm{m}$ behavior, (c) $5 \mu \mathrm{m}$ behavior.

To quantify this separation phenomenon, the forces acting during the separation time were averaged. The force results compared with the separation efficiency curve as shown in the Figure 8. The difference in the equilibrium action of centrifugal force and drag occurs around the diameter with a separation efficiency of $50 \%$. That is, the diameter with the same centrifugal force and drag force with $50 \%$ separation efficiency is the critical diameter that can explain the cyclone particle separation. The critical diameter, in which centrifugal force and drag force act similarly, is an important dependent variable. The critical diameter was selected as a neural network output variable.

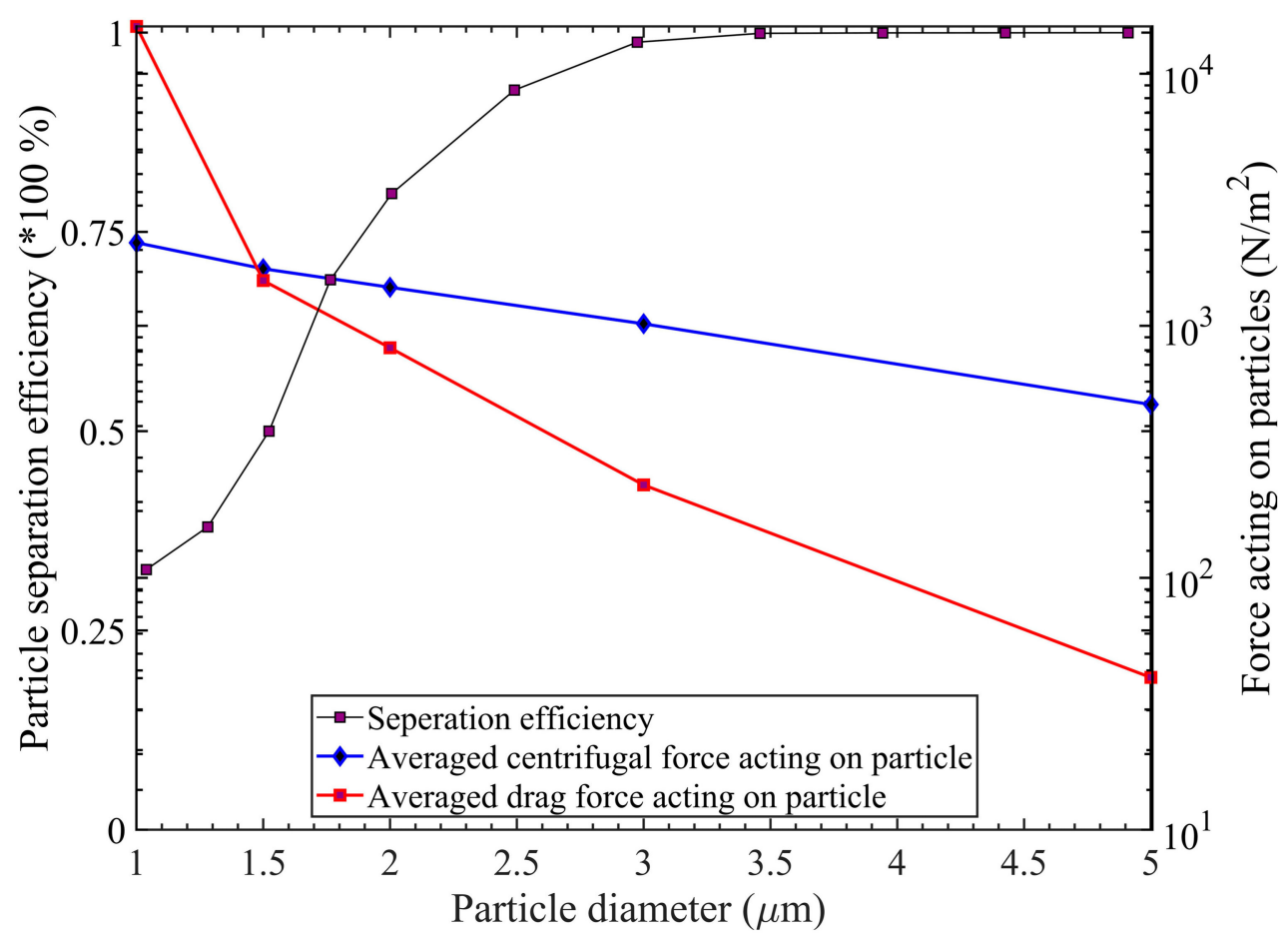

Figure 8. The averaged force results acting during the separation time with the separation efficiency curve. 


\subsection{Cyclone Performance Prediction Model Development Using Neural Network Algorithm}

The neural network method was applied to develop the cyclone critical diameter prediction model. The predictive performance of a neural network algorithm depends on the learning parameters. In order to develop the optimal prediction model, the optimal learning parameters should be obtained. The leaning parameters are Epoch, batch size, and learning rate. The epoch is the number of back propagation process. The batch size is the number of data used for backpropagation process. The learning rate refers to the amount to learn when updating the model's weights. The hyperparameter tuning was performed with random sampling method to derive parameters that affect the prediction model. The number of sampling was set to 500 in order to consider diversity of design space. The result of parameter optimization is shown in the Figure 9. The Figure 9 shows the results predicted by a random combination of learning parameters. The right end of the Figure 9 is a quantification of prediction performance according to parameter combinations. The $\mathrm{R}$ square and mean normalized error were used as indicators for quantitative evaluation of the model. $R$ square represents the degree of agreement between the true value and the predicted value, and the closer to 1 , the higher the performance. The mean normalizes error (MNE) is an index that can objectively evaluate the model's performance. The optimal parameter combinations are summarized in the Table 6.

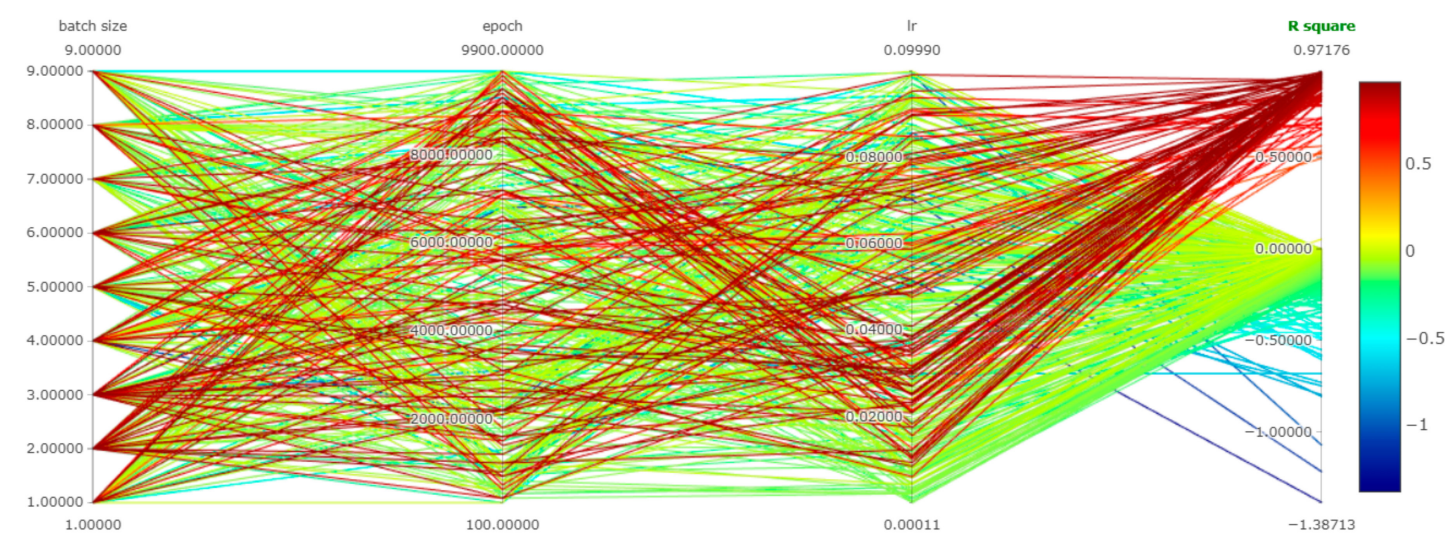

Figure 9. Hyperparameter tuning results.

Table 6. The optimized learning parameters.

\begin{tabular}{cc}
\hline Optimized Parameters & Values \\
\hline Epoch & 5200 \\
Learning rate & 0.00054 \\
Batch size & 2 \\
Number of layer & 5 \\
Node & $8 / 16 / 24 / 16 / 8$ \\
\hline
\end{tabular}

The model was developed using the train set based on the optimal learning parameter combination, and the performance of the neural network model was evaluated using the test set. To evaluate the predictive performance of the neural network model, the results of multi linear regression as reprehensively traditional surrogate model were compared. The result of comparing the prediction performance of the multi linear regression (MLR) model and the neural network model was shown in the Figure 10. Unlike the MLR model, since the neural network model can create a complex nonlinear relationship between the cyclone design variable and the critical diameter, it shows better predictive performance than the traditional method. The results of expressing this quantitatively are shown in the Table 7. The Neural network (NN) results increased about $32.2 \%$ and $27.6 \%$ in 22 and MNE, respectively, compared to MLR. This shows very good prediction performance. Figure 11 represents the training results and prediction results by the NN and MLR, respectively. 


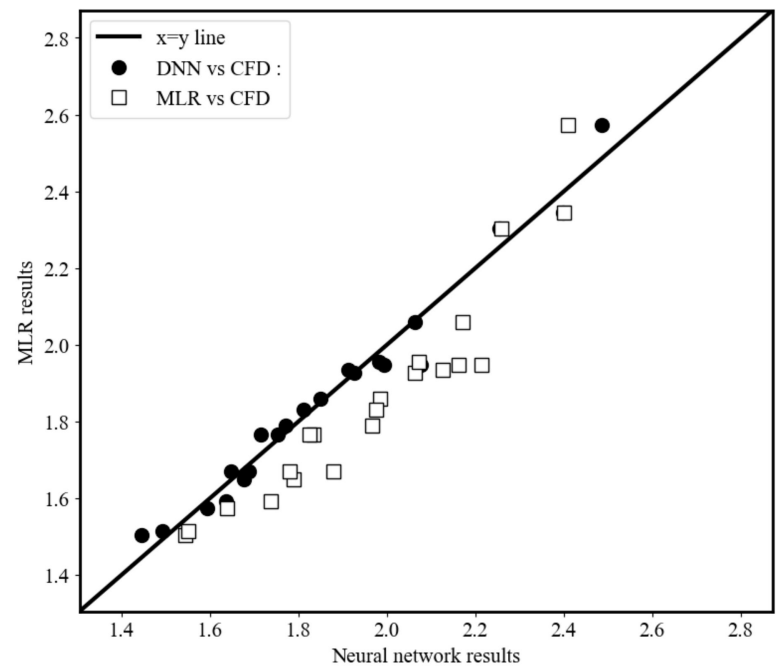

Figure 10. The result of comparing the prediction performance of the MLR model and the neural network model.

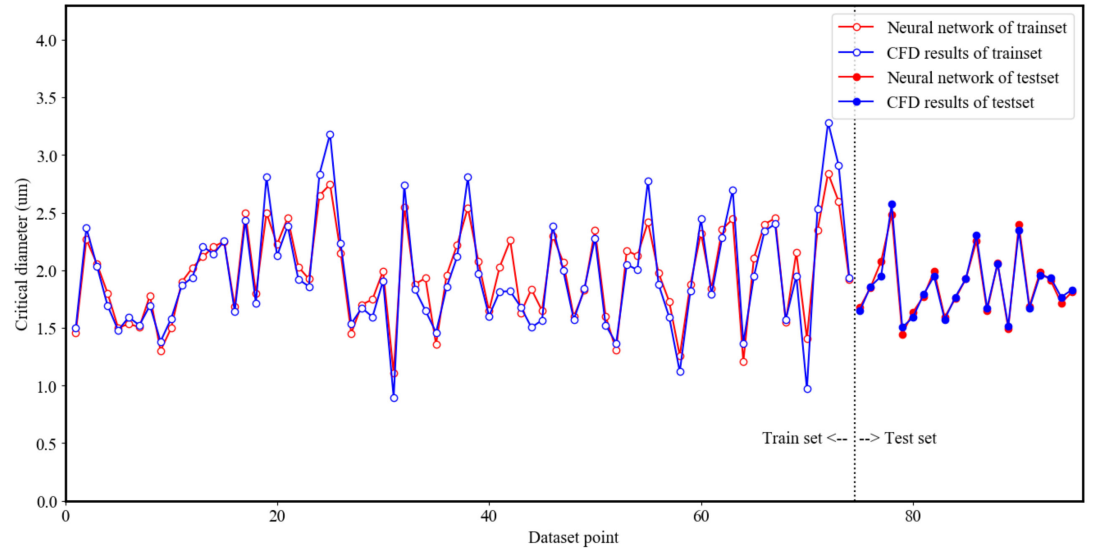

(a)

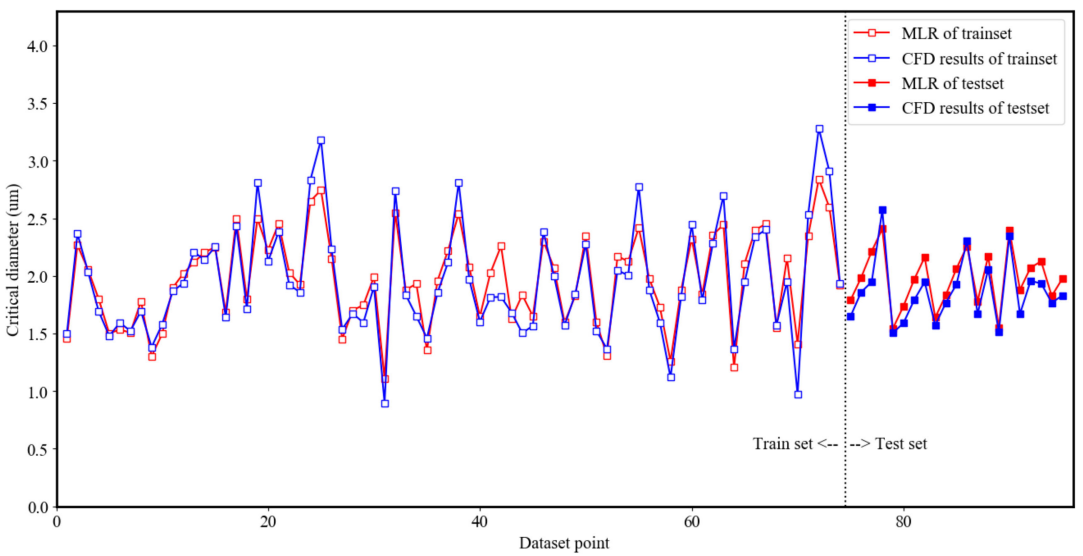

(b)

Figure 11. The training results and prediction results by the NN and MLR; (a) Neural network results; (b) Multi linear regression.

Table 7. Neural network prediction model performance comparing with MLR results.

\begin{tabular}{cccc}
\hline Metric & MLR & NN & Improvement \\
\hline Mean normalized error & 6.73 & 1.86 & $-27.6 \%$ \\
$\mathrm{R}^{2}$ & 0.735 & 0.972 & $+32.2 \%$ \\
\hline
\end{tabular}




\section{Conclusions}

In this paper, the characteristics of the cyclone separator from the Lagrangian perspective to design important dependent variables, develops a neural network model for predicting the separation performance parameter. The conclusion can be drawn as follow:

(1) The particle behavior characteristics in the cyclone were analyzed from the Lagrangian perspective. It was demonstrated that the centrifugal force and the drag force are similar in the diameter with the $50 \%$ separation efficiency. This indicates that the critical diameter is important dependent variable for cyclone design based on particle separation theory. Therefore, the critical diameter was applied to the neural network as the design dependent variable.

(2) The neural network model was developed by using CFD combinations that considered various design space based on the DoE. The learning parameters of developed model showed sufficient distribution in the design space, and the neural network prediction model can accurately predict the critical diameter obtained by CFD. Furthermore, the neural network prediction results showed superior performance compared to the traditional multi linear regression results. Therefore, the CFD methodology combined with the neural network method can be applied for efficient and fast design of the cyclone.

In a future study, we plan to find a wider design area point based on the critical diameter ANN model and global optimization algorithm, or derive the optimal critical diameter, and investigate the generalization characteristics of the neural network model through experimental method.

Author Contributions: D.P. and J.S.G. analyzed the numerical data; D.P. developed the neural network model and organized draft paper. All authors have read and agreed to the published version of the manuscript.

Funding: This research was financially supported by the National Research Foundation of Korea (NRF) grant funded by the Korea government (MOE) (No. NRF-2017R1A2B2006264).

Conflicts of Interest: The authors declare no potential conflict of interest with respect to the research, authorship and publication of this article.

\section{References}

1. Iozia, D.L.; Leith, D. Effect of cyclone dimensions on gas flow pattern and collection efficiency. Aerosol Sci. Technol. 1989, 10, 491-500. [CrossRef]

2. Shepherd, C.B.; Lapple, C.E. Flow Pattern and Pressure Drop in Cyclone Dust Collectors: Cyclone without Inlet Vane. Ind. Eng. Chem. 1940, 32, 1246-1248. [CrossRef]

3. Avci, A.; Karagoz, I. Theoretical investigation of pressure losses in cyclone separators. Int. Commun. Heat Mass Transf. 2001, 28, 107-117. [CrossRef]

4. Raoufi, A.; Shams, M.; Farzaneh, M.; Ebrahimi, R. Numerical simulation and optimization of fluid flow in cyclone vortex finder. Chem. Eng. Process. Process Intensif. 2008, 47, 128-137. [CrossRef]

5. Wang, B.; Xu, D.L.; Chu, K.W.; Yu, A.B. Numerical study of gas-solid flow in a cyclone separator. Appl. Math. Model. 2006, 30, 1326-1342. [CrossRef]

6. Misiulia, D.; Andersson, A.G.; Lundström, T.S. Chemical Engineering Research and Design Effects of the inlet angle on the flow pattern and pressure drop of a cyclone with helical-roof inlet. Chem. Eng. Res. Des. 2015, 2, 307-321. [CrossRef]

7. Bogodage, S.G.; Leung, A.Y.T. CFD simulation of cyclone separators to reduce air pollution. Powder Technol. 2015, 286, 488-506. [CrossRef]

8. Elsayed, K.; Lacor, C. Numerical modeling of the flow field and performance in cyclones of different cone-tip diameters. Comput. Fluids 2011, 51, 48-59. [CrossRef]

9. De Souza, F.J.; Salvo, R.D.V.; Martins, D.D.M. Effects of the gas outlet duct length and shape on the performance of cyclone separators. Sep. Purif. Technol. 2015, 142, 90-100. [CrossRef]

10. Hamdy, O.; Bassily, M.A.; El-Batsh, H.M.; Mekhail, T.A. Numerical study of the effect of changing the cyclone cone length on the gas flow field. Appl. Math. Model. 2017, 46, 81-97. [CrossRef] 
11. Elsayed, K.; Lacor, C. Modeling and Pareto optimization of gas cyclone separator performance using RBF type artificial neural networks and genetic algorithms. Powder Technol. 2012, 217, 84-99. [CrossRef]

12. Safikhani, H. Modeling and multi-objective Pareto optimization of new cyclone separators using CFD, ANNs and NSGA II algorithm. Adv. Powder Technol. 2016, 27, 2277-2284. [CrossRef]

13. Park, D.; Cha, J.; Kim, M.; Go, J.S. Multi-objective optimization and comparison of surrogate models for separation performances of cyclone separator based on CFD, RSM, GMDH-neural network, back propagation-ANN and genetic algorithm. Eng. Appl. Comput. Fluid Mech. 2020, 14, 180-201. [CrossRef]

14. Sun, X.; Kim, S.; Yang, S.D.; Kim, H.S.; Yoon, J.Y. Multi-objective optimization of a Stairmand cyclone separator using response surface methodology and computational fluid dynamics. Powder Technol. 2017, 320, 51-65. [CrossRef]

15. Safikhani, H.; Hajiloo, A.; Ranjbar, M.A. Modeling and multi-objective optimization of cyclone separators using CFD and genetic algorithms. Comput. Chem. Eng. 2011, 35, 1064-1071. [CrossRef]

16. Elsayed, K.; Lacor, C. CFD modeling and multi-objective optimization of cyclone geometry using desirability function, Artificial neural networks and genetic algorithms. Appl. Math. Model. 2013, 37, 5680-5704. [CrossRef]

17. ANSYS Inc. ANSYS FLUENT Theory Guide; ANSYS FLUENT-16.1; ANSYS Inc.: Canonsburg, PA, USA, 2018.

18. Tang, Y.; Guo, B.; Ranjan, D. Numerical simulation of aerosol deposition from turbulent flows using three-dimensional RANS and les turbulence models. Eng. Appl. Comput. Fluid Mech. 2015, 9, 174-186. [CrossRef]

19. Obermair, S.; Woisetschläger, J.; Staudinger, G. Investigation of the flow pattern in different dust outlet geometries of a gas cyclone by laser Doppler anemometry. Powder Technol. 2003, 138, 239-251. [CrossRef]

20. Obermair, S.; Staudinger, G. The dust outlet of a gas cyclone and its effects on separation efficiency. Chem. Eng. Technol. 2001, 24, 1259-1263. [CrossRef]

21. Siddique, W.; El-Gabry, L.; Shevchuk, I.V.; Fransson, T.H. Validation and Analysis of Numerical Results for a Two-Pass Trapezoidal Channel With Different Cooling Configurations of Trailing Edge. J. Turbomach. 2012, 135, 1-8. [CrossRef]

Publisher's Note: MDPI stays neutral with regard to jurisdictional claims in published maps and institutional affiliations.

(C) 2020 by the authors. Licensee MDPI, Basel, Switzerland. This article is an open access article distributed under the terms and conditions of the Creative Commons Attribution (CC BY) license (http://creativecommons.org/licenses/by/4.0/). 